\title{
A variation of the Stern-Brocot tree
}

\author{
Colin L. Mallows
}

\begin{abstract}
We study of a variation of the Stern-Brocot tree, in which not one but two fractions are inserted between each existing pair. Relating this tree to the original one gives rise to a permutation of the natural numbers.
\end{abstract}

KEYWORDS AND PHRASES: Stern-Brocot tree, permutations of $\mathcal{N}$.

\section{The Stern-Brocot tree, and a variation}

The Stern-Brocot tree (or rather half of it) can be defined as follows. Start with two fractions $0 / 1$ and $1 / 1$, forming an ordered set $S_{0}$. (Throughout this paper, "fraction" means "fraction in lowest terms".) At stage $k$, $(k=1,2, \ldots)$, form a new set $S_{k}$ by inserting between each pair of adjacent fractions in $S_{k-1}$, say $p / q$ and $r / s$, the fraction $(p+r) /(q+s)$. Name the (ordered) set of fractions that are introduced at this stage $R_{k}$. Thus $R_{1}=(1 / 2)$, $R_{2}=(1 / 3,2 / 3), R_{3}=(1 / 4,2 / 5,3 / 5,3 / 4), R_{4}=(1 / 5,2 / 7,3 / 8,3 / 7,4 / 7,5 / 8$, $5 / 7,4 / 5)$ etc. $R_{k}$ has $2^{k-1}$ elements. It is well known (see e.g. [1]) that every proper fraction appears (exactly once) in some $R_{k}$, and that adjacent fractions $p / q, r / s$ satisfy

$$
|q r-p s|=1 .
$$

We define a new tree (first noticed in [2, Section 9]) starting with $S_{0}^{\prime}=$ $S_{0}$. At the $k$-th stage insert two fractions between each existing adjacent pair in $S_{k-1}^{\prime}$, namely between $p / q$ and $r / s$ (where $p$ is even and $r$ is odd), insert $(p+r) /(q+s)$ and $(p+2 r) /(q+2 s)$. Notice that we may have either $p / q<(p+r) /(q+s)<(p+2 r) /(q+2 s)<r / s$ or the same with all the inequalities reversed. It is easy to see that every adjacent pair of fractions in $S_{k}^{\prime}$ satisfy (1) and that the numerators of successive fractions in $S_{k}^{\prime}$ are alternately even and odd, so that the insertion rule is well-defined. Successive generations of insertions are denoted $R_{1}^{\prime}, R_{2}^{\prime}, \ldots$ Thus $R_{k}^{\prime}$ has $2.3^{k-1}$ elements. 
Explicitly,

$$
\begin{aligned}
R_{1}^{\prime}= & (1 / 2,2 / 3), \quad R_{2}^{\prime}=(1 / 3,2 / 5,4 / 7,3 / 5,3 / 4,4 / 5) \\
R_{3}^{\prime}= & (1 / 4,2 / 7,4 / 11,3 / 8,2 / 7,4 / 9,6 / 11,5 / 9,7 / 12,10 / 17,8 / 13,5 / 8,5 / 7 \\
& 8 / 11,10 / 13,7 / 9,5 / 6,6 / 7)
\end{aligned}
$$

Lemma 1. For every proper fraction $x$, there is a $k$ such that $x$ appears in $R_{k}^{\prime}$.

Proof. Define the "ndsum" of a fraction $p / q$ to be $p+q$. An easy induction shows that for $k \geq 1$ the minimum ndsum in the row $R_{k}^{\prime}$ is $k+2$ (attained by the first element, which is $1 /(k+1))$. The minimum ndsum in $S_{0}^{\prime}$ is 1 , attained by $0 / 1$. Suppose the fraction $a / b$, where $a+b \geq 2$, does not appear in any $R_{k}^{\prime}$. Consider the row $R_{a+b}^{\prime}$. There must be a fraction $p / q$ in this row and a fraction $r / s$ in $S_{a+b}^{\prime}$ such that $|q r-p s|=1$ and $p / q<a / b<r / s$, or the same with both inequalities reversed. Suppose the inequalities are as shown. Then $a q-b p>0$, so $a q-b p \geq 1$, and similarly $b r-a s \geq 1$. Thus

$$
(p+q)(b r-a s)+(r+s)(a q-b p) \geq p+q+r+s .
$$

But the l.h.s. of $(2)$ equals $(a+b)(q r-p s)=a+b$, and the r.h.s. is at least $(a+b+2)+1$, so $a+b \geq a+b+3$ which is a contradiction. When the inequalities are reversed, the argument is similar.

\section{Relating the two trees}

We study the relation between the sets $\left\{R_{k}^{\prime}\right\}$ and $\left\{R_{k}\right\}$. We find that (as far as we have computed, namely $R_{6}^{\prime}$ and $R_{12}$ ) there is a sequence $p$, starting

Sequence $p$

$1,2,5,3,4,8,17,9,10,20,11,6,7,14,29,15,16,32,65,33,34,68,35,18,19$,

such that for each $k$, and for $i=1,2, \ldots, 2.3^{k-1}$, the fraction $R_{k}^{\prime}(i)$ appears as $R_{k^{\prime}(i)}(p(i))$ for some $k^{\prime}(i)$. We write $k^{\prime}(i)=k+r_{k}(i)$, and set $n_{k}=2^{k^{\prime}-1}$, which is the sequence of lengths of the rows $k^{\prime}$ of $R$ in which these fractions appear. Thus for $k=3$, the rows of the following matrix $M_{3}$ are

the numerators of fractions in $R_{3}^{\prime}$

the corresponding denominators

the $m$ such that each such fraction appears in $R_{m+3}$

the length of the row $R_{m+3}$ (this is $n_{m+3}$ )

the position of this fraction in $R_{m+3}$ (this is a prefix of $p$ ). 


$\begin{array}{rrrrrrrrrrrrrrrrrr}1 & 2 & 4 & 3 & 3 & 4 & 6 & 5 & 7 & 10 & 8 & 5 & 5 & 8 & 10 & 7 & 5 & 6 \\ 4 & 7 & 11 & 8 & 7 & 9 & 11 & 9 & 12 & 17 & 11 & 8 & 7 & 11 & 13 & 9 & 6 & 7 \\ 0 & 1 & 2 & 1 & 1 & 2 & 3 & 2 & 2 & 3 & 2 & 1 & 1 & 2 & 3 & 2 & 2 & 3 \\ 4 & 8 & 16 & 8 & 8 & 16 & 32 & 16 & 16 & 32 & 16 & 8 & 8 & 16 & 32 & 16 & 16 & 32 \\ 1 & 2 & 5 & 3 & 4 & 8 & 17 & 9 & 10 & 20 & 11 & 6 & 7 & 14 & 29 & 15 & 16 & 32\end{array}$

Rows 3 and 5 of the first six columns of this matrix give the corresponding results for $R_{2}^{\prime}$, while the fourth row is twice the fourth row for $R_{2}^{\prime}$.

We have studied similar matrices through $k=6$, finding that for each $k$, the fifth row of $M_{k}$ contains the first $2.3^{k-1}$ elements of the sequence we have called $p$. The third row contains numbers in the range $(0, k)$, with successive entries equal or consecutive.

The following lemma shows how the sequence for $R_{k+1}^{\prime}$ can be obtained from that for $R_{k}^{\prime}$.

Lemma 2. Given the finite sequences $p_{k}$ and $n_{k}$ that describe the relation of $R_{k}^{\prime}$ to the rows of $S$, the sequences for row $R_{k+1}^{\prime}$ are as follows.

$$
\begin{aligned}
& p_{k+1}=\left(p_{k}, \operatorname{rev}\left(3 n_{k}+1-p_{k}\right), 3 n_{k}+p_{k}\right), \\
& n_{k+1}=\left(2 n_{k}, \operatorname{rev}\left(4 n_{k}\right), 4 n_{k}\right)
\end{aligned}
$$

where "rev" means "the reverse of".

Proof. The $n$ and $p$ sequences for $R_{k+1}^{\prime}$ are unchanged if we replace the starting fractions $S_{0}$ and $S_{0}^{\prime}$ by $(0 / 1,1 / 2)$. So the (finite) $p$ sequence for $R_{k}^{\prime}$ is the same as the first third of the $p$ sequence for $R_{k+1}^{\prime}$, while the rows for $R_{m+1}$ are twice as long as those for $R_{m}$. Similarly, the final third of the $p$-sequence for $R_{k+1}^{\prime}$, which relate to the interval $(2 / 3,1 / 1)$, are the same as the sequence for $R_{k}^{\prime}$, translated by $3 / 4$ of the length, which is four times the length for $R_{m}$. Finally, for the middle third, which relates to the interval $(1 / 2,2 / 3)$, we have to read the $R_{k}$ values backwards (because the numerator of $1 / 2$ is odd and the numerator of $2 / 3$ is even) and count backwards from $3 / 4$ of the lengths.

This lemma makes it easy to compute $p$ as far as desired. However it has not led us to a proof that the sequence $p$ is a permutation of the natural numbers. We will show that another sequence, $p p$, which we have checked agrees with $p$ through 354,294 terms, is indeed a permutation.

\section{The sequences $b$ and $p p$}

To approach the sequence $p p$, we must first define another sequence $b(\mathcal{N})$. 
Algorithm B. $b(1)=1$. For $k \geq 1$,

$$
(b(3 k-1), b(3 k), b(3 k+1))=(4 i-1,2 i, 4 i+1)
$$

where $i=b(k)$. Thus the sequence $b$ begins

$$
1,3,2,5,11,6,13,7,4,9,19,10,21,43,22,45,23,12,25,51 \text {, }
$$$$
26,53,27,14,29, \ldots
$$

Theorem 1. The sequence $b(\mathcal{N})$ is a permutation of $\mathcal{N}$.

Proof. Suppose $m$ is the smallest integer that does not appear as an element of $b(\mathcal{N})$. It is impossible that $m$ is even, since $m / 2$ does appear, and for some $k$ we have $b(k)=m / 2$. Then $m$ must appear at $b(3 k)$. If $m$ is odd, set $i=\operatorname{round}(m / 4)$. Then $i$ appears at some point $k, b(k)=i<m$, so that $m$ appears as an element of the triad centered at $3 k$. Thus all integers must appear. A similar argument shows that no integer can appear twice. Suppose $m$ is the smallest integer that appears twice. If $m$ is even, we have $b\left(3 k_{1}\right)=b\left(3 k_{2}\right)=m$, with $k_{1} \neq k_{2}$. Then $b\left(k_{1}\right)=b\left(k_{2}\right)=m / 2$ so that the integer $m / 2$ appears twice before $m$ does. Thus $m$ cannot be even. If $m$ is odd, suppose first that the smallest violation is $b\left(3 k_{1}-1\right)=b\left(3 k_{2}-1\right)=$ $4 i-1$, with $k_{1} \neq k_{2}$. Then $b\left(3 k_{1}\right)=b\left(3 k_{2}\right)=2 i$ so that $b\left(k_{1}\right)=b\left(k_{2}\right)=i$, and $i$ appears twice before $4 i-1$ does. Similarly if $m=4 i+1$.

We define another sequence $p p$ by:

Algorithm PP. $p p(1)=1, p p(2)=2$. For $k=1,2, \ldots$

$$
(p p(4 k-1), p p(4 k), p p(4 k+1), p p(4 k+2))=(6 i-1,3 i, 3 i+1,6 i+2)
$$

where $i=b(k)$.

Theorem 2. The sequence $p p(\mathcal{N})$ is a permutation of $\mathcal{N}$.

Proof. Since the sequence $b$ is a permutation of $\mathcal{N}$, it is clear that numbers of the form $3 i$ and $3 i+1$ appear just once in $p p$, in positions $4 k$ and $4 k+1$, and numbers of the form $3 i-1$ appear in positions $4 k-1$ and $4 k+2$, where $i=b(k)$.

We have verified that the sequences $p$ and $p p$ agree through their first 354,294 terms. Of course, this result does not prove anything about the sequence $p$, merely that it agrees with the facts as far as we have computed them. We have not been able to prove that Algorithms P and PP generate the same sequence. 
We think it remarkable that (it appears) Algorithm B and Algorithm P are so closely related, since $b$ generates blocks of length 4 in PP, while Algorithm $\mathrm{P}$ generates the sequence $p$ in blocks of length $2,4,12,36, \ldots$ with the first half of each block involving reading previous blocks backwards.

\section{Generalizations}

Once we have the sequence $b$ in hand, we can generate many permutations of $\mathcal{N}$ by constructions similar to that in Algorithm PP. For example,

Algorithm AA. $a a(1,2,3)=(1,2,3)$. For $k \geq 1$,

$$
\begin{aligned}
& (a a(6 k-2), a a(6 k-1), a a(6 k), a a(6 k+1), a a(6 k+2), a a(6 k+3)) \\
& \quad=(8 i-2,8 i-1,4 i, 4 i+1,8 i+2,8 i+3)
\end{aligned}
$$

where $i=b(k)$.

This particular sequence happens to be identical to one that makes no reference to the sequence $b$, but is generated by the following

Algorithm A. Set $a(1)=1$. For $n \geq 1$ :

(5) $a(2 n+1)=1+a(2 n)$.

The proof of this equality is left for another occasion.

There are other ways of defining a modified Stern-Brocot tree, but we have not found any as elegant as the one we have presented.

\section{References}

[1] Graham, R.L., Knuth, D.E. and Patashnik, O. (1994). Concrete Mathematics. Addison-Wesley, Boston, MA. MR1397498

[2] Guettler, G. and Mallows, C. (2010). A generalization of Apollonian packing of circles. J. Combinatorics 1 1-27. MR2675919 
Colin L. Mallows

AvaYa Labs

BASKING RIDGE

NJ 07920

USA

E-mail address: colinm@research.avayalabs.com

Received October 10, 2010 